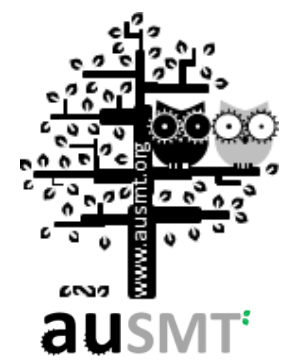

\title{
Stair Climbing in a Quadruped Robot
}

\author{
Shen-Chiang Chen, Chih-Chung Ko, Cheng-Hsin Li, \\ and Pei-Chun Lin* \\ Department of Mechanical Engineering, National Taiwan University \\ (Received 30 March 2011; Accepted 30 August 2011; Published on line 1 March 2012) \\ *Corresponding author: peichunlin@ntu.edu.tw \\ DOI: 10.5875/ausmt.v2i1.77
}

\begin{abstract}
This paper reports the algorithm of trajectory planning and the strategy of four-leg coordination for quasi-static stair climbing in a quadruped robot. This development is based on the geometrical interactions between robot legs and the stair, starting from single-leg analysis, followed by two-leg collaboration, and then four-leg coordination. In addition, a brief study on the robot's locomotion stability is also included. Finally, simulation and experimental testing were executed to evaluate the performance of the algorithm.
\end{abstract}

Keywords: Quadruped; Stair climbing; Trajectory planning; Leg coordination

\section{Introduction}

Locomotion of robots on uneven terrain has drawn great attention in recent years. It has been widely reported that robots can achieve great mobility on rough terrains. However, much less research has been conducted related to stair climbing. Some examples of stair-climbing robots include the Shrimp Rover [1], which has a clever mechanism design that combines wheels and self-adjustable linkages to maintain suitable body posture and increase its mobility on uneven terrain and stairs. Similarly, Loper [2] climbs stairs by rotating four Tri-lobe wheels. IMPASS [3] climbs obstacles using two rimless spoke wheels with two degrees of freedoms (DOFs). Some tracked robots [4] also utilize the treads on the tracks, which on certain levels can grab the edges of stairs. The humanoid robot ASIMO [5], developed by HONDA, has demonstrated stair climbing behavior quite frequently in various robot shows. The hexapod ASTERISK [6] climbs stairs based on precise recognition of each stair by laser scanner. The hexapod robot RHex [7] also demonstrates excellent performance on stairs, including both stair ascent [8] and descent [9]. As for the quadrupeds, several of them are reported to have great mobility; for example, the Scout series [10], Tekken [11], Titan [12], and RIMHO [13] climb stairs utilizing various forms of sensory feedback, such as contact sensors, inclinometers, and joint sensors. Recently, the DARPA Learning Locomotion Program, which uses the BDI-developed small quadruped robot Little Dog as a common platform, included stair climbing as one of its tasks. However, due to its small size compared to the stair, the gait developed for this platform usually performs in an intermittent manner. In general, however, literature about stair climbing by quadrupeds is still very limited.

In addition to our goal of driving Quattroped [14] on various terrains, we are interested in investigating general stair climbing strategy in a mid-size quadruped (i.e. body length around 40-80 cm), which is approximately the minimum size of a robot capable of continuous stair climbing. Because the rolling locomotion induced by the half-circle legs of Quattroped is not typical, we are focusing on the more general "point contact" legs to construct a basic framework and address how to treat the stair climbing problem from kinematic and geometrical point of views. The algorithm developed for this purpose requires various assumptions but was not designed for a particular robot, so it might be suitable for a larger family of mid-size quadrupeds. 


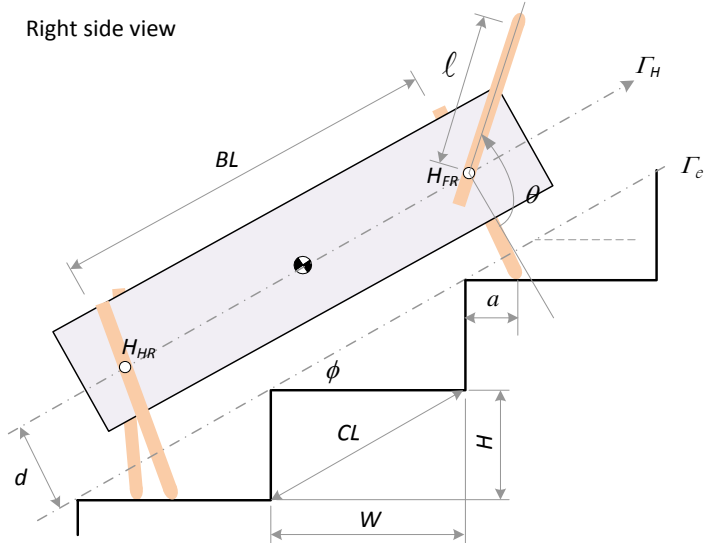

Figure 1. Illustrative drawing of a quadruped that climbs stairs.

The next section introduces relevant terminologies and assumptions utilized in the algorithm, followed by trajectory planning, which is detailed in the section on trajectory planning, including analysis of a single leg, coordination of two and four legs, and a brief summary of trajectory planning procedure. A section on stability analysis briefly investigates the quasi-static stability of a robot during stair climbing, followed by simulation results. A section on error adoption briefly reviews the error sources. This is followed by a section of experiment results reporting and discussing the field test results. The last section concludes the work.

\section{Terminologies and Assumptions}

The goal of this paper is to construct a framework with a steady stair climbing gait in a quadruped robot, mainly including leg trajectory generation and 4-leg coordination. The design is created in work space then transformed into joint space for robot locomotion.

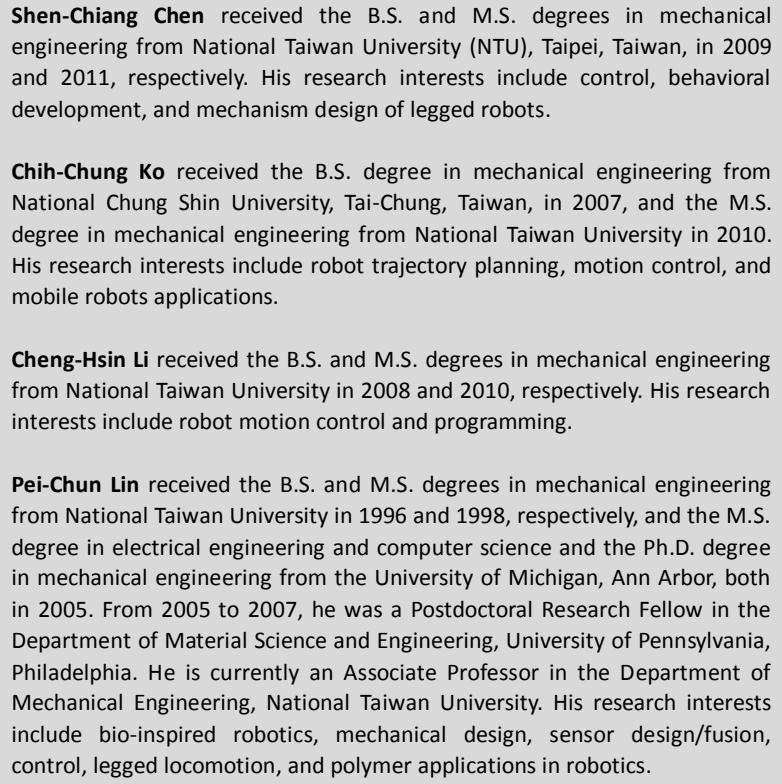

Chih-Chung Ko received the B.S. degree in mechanical engineering from National Chung Shin University, Tai-Chung, Taiwan, in 2007, and the M.S degree in mechanical engineering from National Taiwan University in 2010. His research interests include robot trajectory planning, motion control, and mobile robots applications.

Cheng-Hsin Li received the B.S. and M.S. degrees in mechanical engineering from National Taiwan University in 2008 and 2010, respectively. His research interests include robot motion control and programming.

Pei-Chun Lin received the B.S. and M.S. degrees in mechanical engineering from National Taiwan University in 1996 and 1998, respectively, and the M.S. degree in electrical engineering and computer science and the Ph.D. degree in mechanical engineering from the University of Michigan, Ann Arbor, both in 2005. From 2005 to 2007, he was a Postdoctoral Research Fellow in the Department of Material Science and Engineering, University of Pennsylvania, Philadelphia. He is currently an Associate Professor in the Department of Mechanical Engineering, National Taiwan University. His research interests include bio-inspired robotics, mechanical design, sensor design/fusion, control, legged locomotion, and polymer applications in robotics.

Assumptions and associated terminologies used in the algorithm are listed below and depicted in Figure 1:

1. Stair: Characteristic length $C L$ and slope of the stair are defined as $C L=\sqrt{W^{2}+H^{2}}$ and $\phi=\arctan (H / W)$, respectively, where $W$ and $H$ are width and height of each step. Empirically measured nominal value of $W$ and $H$ of domestic stairs are 27 $\mathrm{cm}$ and $17 \mathrm{~cm}$, accordingly, and the standard deviations of $W$ and $H$ are $2.8 \mathrm{~cm}$ and $1.2 \mathrm{~cm}$ respectively.

2. Individual leg motion: Each leg $L_{i,}, i=F R, F L, H R, H L$ is required to be capable of two degrees-of-freedom (DOF) planar motion in the sagittal plane. The subscripts $F R, F L, H R$, and $H L$ denote front right, front left, hind right, and hind left legs, respectively. For simplicity, the foot position of each leg will be represented in the polar coordinate $(\ell, \theta)$ with its origin located at the hip joint of each leg $H_{i, i=F R, F L, H R, H L}$. It should also be noted that the 2 DOF articulated leg is also compatible with the following development, since its joint angles $\left(\theta_{1}, \theta_{2}\right)$ can be transformed into the polar coordinates using straight-forward trigonometric operations.

3. Leg arrangement: Front/back hip joints of right and left legs $\left(H_{F R}\right.$ and $H_{F L} / H_{H R}$ and $\left.H_{H L}\right) \quad$ coincide at the same point from a side view. Body length $B L$ is defined as the distance between the front hip joint $\left(H_{F R}\right.$ or $\left.H_{F L}\right)$ and the hind hip joint $\left(H_{H R}\right.$ or $\left.H_{H L}\right)$.

4. Robot motion: The robot body is modeled as a rectangle, and its center of mass (COM) is assumed to be located at the center of the rectangle. The robot body is assumed to be operated in the quasi-static constant-velocity forward motion without any pitch and roll motion, and dynamics of the swing leg are ignored due to its low inertia compared to that of the body. In addition, the foot is the only portion of the robot to make ground contact during locomotion, like that of quadruped animals in general. These assumptions have the following implications:

(1) The trajectories of the front and hind hip joints $\left(H_{F R} / H_{F L}\right.$ and $\left.H_{H R} / H_{H L}\right)$ can be moved with the same trajectory $\Gamma_{H}$ with an offset distance $d$ from the line connected by the edges of the steps $\Gamma_{e}$ as shown in Figure 1 ( $d$ hereafter referred to as hip clearance). In addition, the robot body also inclines with the same slope as the stair $\phi$, and the trajectory of the COM moves with the same trajectory $\Gamma_{H}$ as well.

(2) At least three feet touch the steps at the same time to maintain static stability (i.e. at most one leg is allowed to swing in the air at any time). 
(a)

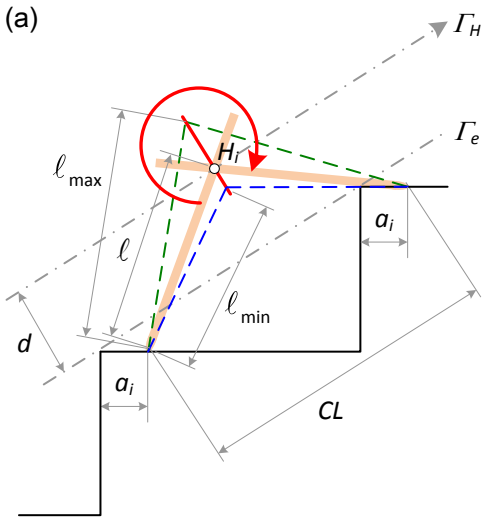

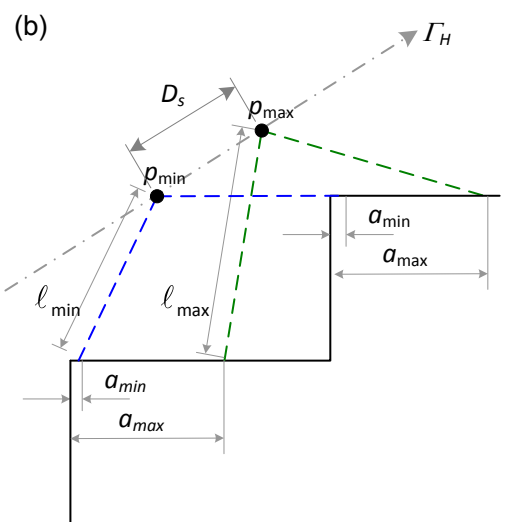

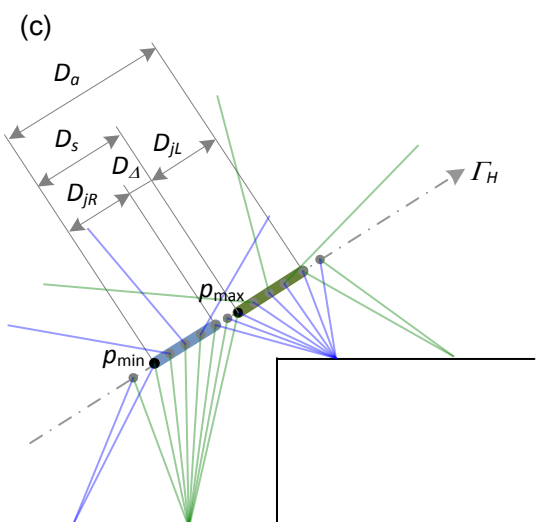

Figure 2. (a) Configurations of a leg "right before" the lift-off of the foot from the lower step (left black bar) and "right after" the touchdown on the upper step (right black bar). (b) Schematic diagram of two extremes: the earliest timing (blue line) and the latest (green line) timing to start swinging the leg from the lower step to the upper step. (c) Illustrative drawing that shows swings of the left and right legs from the lower step to the upper step.

(3) Geometrically, the stair is the periodic composition of steps with a certain width and height. Thus, the nominal trajectory of each foot is scheduled to be moved in a periodic manner from one step to another step. For simplicity, the distance between the foot ground-contact point $C_{i, i=F R, F L, R R, R L}$ and the edge of the step, $a_{i, i=F R, F L, R R, R L}$, is assumed fixed (i.e. "periodic 1 " motion; $a_{i}$ is hereafter referred to as contact offset).

\section{Trajectory Planning}

\section{Trajectory analysis of a single leg}

Figure 2(a) depicts the geometrical configurations of a leg $L_{i, i=F R, F L, H R, H L}$ "right before" the lift-off of the foot from the lower step (left pink bar) and "right after" the touchdown on the upper step (right pink bar) with the given arbitrary $a_{i}$. To simplify the design process, we temporarily assume that the leg lengths at the lift-off and the touchdown are equal, and the time duration for the leg to swing from lift-off to touchdown is infinitesimal. These assumptions add conservative constraints on the estimation of the required leg length, and those will be released in the following sections to meet real situations. To avoid collision between the non-foot portion of the leg and the edge of the step during leg swing from the lower step to the upper step, the length of the leg $\ell$ needs to be long enough to lift the hip joint $H_{i, i=F R, F L, H R, H L}$ above the horizontal surface of the upper step, as shown in Figure 2(a). The right blue-dashed line indicates the lowest configuration of the leg $L_{i}$ to meet this requirement. Figure 2(a) clearly shows that $\ell_{\min }$ is purely determined by the geometry of the stair,

$$
\ell_{\min }=\frac{C L}{2} \sec \phi=\frac{W^{2}+H^{2}}{2 W}=\frac{1}{2}(W+H \tan \phi) .
$$

In the meantime, the contact offset $a_{i}$ is constrained as well,

$$
a_{i} \leq \ell_{\min }
$$

and this inequality keeps the hip joint from hitting the stair. Please note that $\ell_{\min }$ does not mean the minimum length the leg can achieve, but the minimum length the leg should have in order to perform a successful swing from the lower step to the upper step. With the empirically-measured nominal values of the stair $W=27 \mathrm{~cm}$ and $H=17 \mathrm{~cm}$, the $\ell_{\text {min }}$ is calculated $18.85 \mathrm{~cm}$. In addition, in general the robot has a maximum operable leg length $\ell_{\max }$ (green dashed line). These two constraints provide upper and lower bounds for feasible leg length during stair climbing $\ell_{\min } \leq \ell \leq \ell_{\max }$, and these two further confine the possible locations of hip joint $H_{i, i=F R, F L, H R, H L}$ on the red line as shown in Figure 2(a). The figure also clearly shows that the longer red line indicates the wider range of the hip clearance $d$ we can select. Figure 2(a) also reveals that the contact offset $a_{i}$ is closely related to $d$, the geometrical configuration of the steps $(C L$ and $\phi)$, and the length of the robot leg $\ell$ in the following manner:

$$
a_{i}=\left(\sqrt{\ell^{2}-\frac{1}{4} C L^{2}}-d\right) \csc \phi .
$$

When the leg length equals its minimum required value $\ell_{\text {min }}$, Equation (3) can be simplified to:

$$
a_{i}=\ell_{\min }-d \csc \phi,
$$


where $a_{i}$ reaches its maximum and equals to $\ell_{\min }$ shown in (2) when $d$ approaches 0 . In general, hip clearance $d$ is an active variable for which we have a preferred value. Thus, combining $d$ with a possible range of leg length $\ell_{\min } \leq \ell \leq \ell_{\max }$, the feasible range of contact offset $a_{i}$ can be derived by (3). This shows that the value $a_{i}$ can be varied with the given maximum leg length $\ell_{\max }$ and the hip clearance $d$. This reveals two trends: First, if the robot's leg has a larger $\ell_{\max }$, a wider range of $a_{i}$ is possible. Second, to have a larger hip clearance $d$ implies the use of a smaller contact offset $a_{i}$, which means the foot should make contact with the step closer to the step edge. In addition, the feasible range of $a_{i}$ also determines the difference between the timings for right and left legs when swinging from the lower step to the upper one, which further determines whether or not coordination among legs is feasible. The details of the related materials will be illustrated in the following section.

\section{Coordination between two front or hind legs}

If only a single leg, with the assumptions defined in the previous section, is considered, the leg is capable of moving from one step to another step as long as the length of the leg is longer than the minimum required length $\ell_{\min }$. However, this requirement is not sufficient if the motions of two front or hind legs are considered together, due to the fact that two legs cannot swing simultaneously in order to maintain the static stability of the robot (i.e. at least three legs need to be on the ground). Since the hip joints of the right and left legs coincide at the same point from a side view as shown in Figure 2(a) $\left(H_{F R}\right.$ and $H_{F L} / H_{H R}$ and $\left.H_{H L}\right)$, different timings for right and left legs also mean different geometrical locations of the hip joints $H_{i}$ with respect to the stair when the right or left leg swings. Thus, with given maximum leg length $\ell_{\max }$ and hip clearance $d$, the feasible positions of $H_{i}$ for leg swing is shown in Figure 2(b). The earliest timing occurs when hip joint $H_{i}$ reaches the point $p_{\min }$ (at which point the leg length is equal to $\ell_{\min }$ ), and the latest timing is when it reaches $p_{\max }$ (at which point the leg length is equal to $\left.\ell_{\max }\right)$. Please note that the contact offset $a_{i}$ is also at its two extremes $a_{\text {min }}$ and $a_{\max }$ with hip joints arriving at $p_{\min }$ and $p_{\text {max }}$. This figure also clearly reveals that longer $\ell_{\max }$ allows for a bigger difference in the configurations (or timings) for the swinging of the right and left legs. In practice, the motor has a torque limit and it is not feasible for a motor to drive a leg to different configurations within infinitesimal timing. Thus, certain position differences between $p_{\min }$ and $p_{\max }$ must exist, so the swing motion can be practically achieved. Though wider separation of $p_{\min }$ and $p_{\max }$ can reduce the requirement of the motor torque, the separation also has an upper limit due to the possible interference of coordination among all four legs, and this effect will be described in detail in the following section.

As previously mentioned, the timing of the leg swing is strongly determined by the position of the hip joint $H_{i}$ relative to the step. Once the hip joint reaches a certain position along the motion trajectory $\Gamma_{H}$, the associate leg $L_{i}$ is required to initiate the swing motion, as depicted in Figure 2(b). If the leg starts to swing earlier than it should, it may hit the edge of the stair (for the blue leg only) or may not be able to reach the upper stair at the designated location with contact offset $a_{i}$ due to length constraint. However, if the leg doesn't start to swing at the necessary location, it may not be able to reach the new contact at the upper stair in time. Both cases will cause instability in the robot's locomotion. Thus, how to arrange adequate timings to swing all four legs in sequence within one characteristic length $C L$ is the essential task for stable robot locomotion.

In reality, the swing of a leg from lift-off position at the lower step to touchdown position at the upper step takes a certain amount of time due to the limitation of the motor torque. Thus, the positions of hip joint $H_{i, i=F R, F L, H R, H L}$ at lift-off and at touchdown will be different on the trajectory $\Gamma_{H}$ based on the assumption of constant-velocity robot locomotion, as described in Section II. Figure 2(c) depicts several snapshots of leg motions during swings, and the green region illustrates the traveling distance $D_{i, i=F R, F L, H R, H L}$ of hip $H_{i}$ for the leg $L_{i}$ to complete a swing. It is clear that the timing to lift-off for the left leg (blue color) cannot happen before the touchdown of the right leg (green color) in order to maintain static stability. On the safe side, a buffer time $D_{\Delta}$ is also designed to avoid any incorrect coordination due to a motion delay.

Following the assumptions shown in Figure 2(b) that the right leg starts to swing at its earliest time $(\ell=$ $\ell_{\min }$, blue color) and the left leg swings at its latest time ( $\ell=\ell_{\max }$, green color), the position (or time) difference $D_{s}$ at which the right and left leg start to swing can be calculated quantitatively:

$$
D_{s}=\frac{W}{H} \sqrt{\ell_{\max }^{2}-\frac{1}{4}\left(W^{2}+H^{2}\right)}-\frac{1}{2} \sqrt{W^{2}+H^{2}}
$$

Assuming all legs have equal $D_{i}$, and $p$ percent of time is reserved as the buffer period $D_{\Delta}$ (i.e. $p_{\Delta}=D_{\Delta} D_{s}$ ) between the right and left $\left(D_{i}\right) \mathrm{s}\left(D_{j R}\right.$ and $\left.D_{j L, j=F, H}\right)$ shown in Figure 2(c), the duration for each leg to swing $D_{i}$ can be computed as 


$$
\begin{aligned}
D_{i} & =(1-p) D_{s} \\
& =(1-p)\left[\frac{W}{H} \sqrt{\ell_{\max }^{2}-\frac{1}{4}\left(W^{2}+H^{2}\right)}-\frac{1}{2} \sqrt{W^{2}+H^{2}}\right]
\end{aligned}
$$

and the complete duration $D_{a}$ of left and right leg pair can be derived as

$$
\begin{aligned}
D_{a} & =(2-p) D_{s} \\
& =(2-p)\left[\frac{W}{H} \sqrt{\ell_{\max }^{2}-\frac{1}{4}\left(W^{2}+H^{2}\right)}-\frac{1}{2} \sqrt{W^{2}+H^{2}}\right] .
\end{aligned}
$$

Please note that the swings of the right and left legs of front or hind leg pairs $\left(D_{F R}\right.$ and $D_{F L}$ or $D_{H R}$ and $\left.D_{H L}\right)$ are set to be executed contiguously. It is not feasible to swing the legs in the sequence of front-hind-front-hind legs because of possible collision with the edge of the step and leg length constraint $\ell_{\max }$ as depicted in Figure 2(c). If $\ell_{\max }$ is much larger than $\ell_{\min }$ and if $d$ is far from the stair, this constraint can be released. In this case the robot size is generally much larger compared to that of the steps.

\section{Coordination among all four legs}

Stair climbing from step to step is a periodic motion, so it is intuitive that all four legs are required to complete the swings while the hip joints or the robot body climbs one step of the stairs (i.e., one $C L$ ). In addition, since right and left legs should swing consecutively, it is more convenient to analyze 4-leg coordination by considering the arrangement of two $D_{a} \mathrm{~s}$ in one $C L$ : one for front legs and one for hind legs. Thus, it is intuitive that $D_{a}$ should be no more than half of $C L$, or it is not possible to put two $\left(D_{a}\right) \mathrm{s}$ in one $C L$, and this also means that a swing of all four legs cannot be done in one step of stair climbing.

The adequate arrangement of two $\left(D_{a}\right) \mathrm{s}$ in one $C L$ depends on two parameters: one is the duration of $D_{a}$, and the other is the body length $B L$. The starting point $p_{\min }$ of $D_{a}$ is located at a specific location with respect to the step, so the freedom to tune the duration of $D_{a}$ lies in the selection of $\ell_{\max }$ which determines the end point $p_{\text {max }}$. After a specific $D_{a}$ is selected, whether two $D_{a} \mathrm{~s}$ overlap with each other is strongly determined by $B L$. Figure 3 shows three different scenarios: In (a) four legs can swing periodically and sequentially without any problem, but in (b) and (c) there exists certain interference between the front leg and hind legs. In Figure 3 , the positions of the hind hip joints $H_{H R} / H_{H L}$ in all three scenarios are aligned with the position $p_{1}$, where the hind legs are required to start the swing phase. In the meantime, the front hip joints $H_{F R} / H_{F L}$ are located at $q_{1}$.

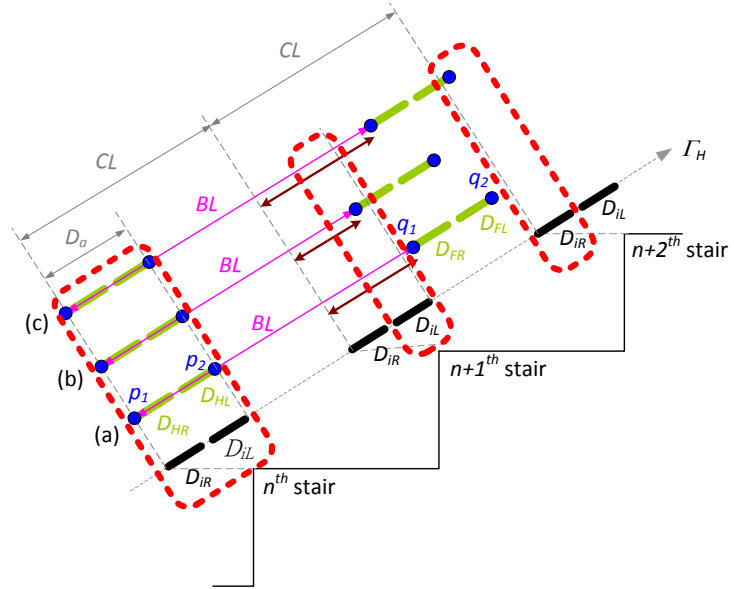

Figure 3. Three typical scenarios of 4-leg coordination: (a) four legs can swing from the lower step to the upper step periodically and sequentially without any problem. (b) Interference - the hind right leg needs to start its swing while the front left leg is still in the swing phase. (c) Interference - the front right leg needs to start its swing while the hind left leg is still in the swing phase. Brown lines indicate rem $(B L, C L)$ used in (8) and (9).

The swing phase of the hind legs ends when the hind hip joints $H_{H R} / H_{H L}$ arrive at $p_{2}$, while the front joints arrive at $q_{2}$. Therefore, it is obvious that in (a) during the entire swing phase $p_{1}$ to $p_{2}$ of the hind legs, the front hip moves from $q_{1}$ to $q_{2}$, and thus is not in the swing phase; so, the adequate swings of all four legs is possible. However, (b) indicates that the hind right leg needs to start its swing (arriving $p_{1}$ ) while the front left leg is still in swing phase, and $(c)$ indicates that the front right leg needs to start its swing before the hind left leg is finished. Both (b) and (c) have certain time periods where two legs are needed in the swing phase; thus the assumption of static stability cannot be maintained thoroughly in one $C L$ motion cycle. The quantitative criteria to avoid interference among 4 legs like (b) and (c), as shown in Figure 3, can be summarized as follows:

$$
\begin{aligned}
& \operatorname{rem}(B L, C L)+D_{a} \leq C L \\
& \operatorname{rem}(B L, C L) \geq D_{a},
\end{aligned}
$$

where $\operatorname{rem}(x, y)$ represents the function to acquire the remainder of $x / y$, and its length is plotted using a brown color in Figure 3 . In addition, the constraint that grants the nonzero duration $D_{a}$ is:

$$
\ell_{\max }>\ell_{\min }
$$

These inequalities represent important relations among dimensions of the stairs, $W$ and $H$, and lengths of the robot body $B L$ and legs $\ell$. Assuming the body length is designed within the range $C L<B L<2 C L$, Equations (8)-(10) can further be simplified as: 


$$
\begin{aligned}
& (B L-C L)+D_{a}\left(W, H, \ell_{\max }, p\right) \leq C L \\
& (B L-C L) \geq D_{a}\left(W, H, \ell_{\max }, p\right) \\
& \ell_{\max }>\left(W^{2}+H^{2}\right) / 2 W .
\end{aligned}
$$

These inequalities are constructed using four major independent variables $\left(W, H, \ell_{\max }\right.$, and $\left.B L\right)$ and one minor variable $p$. Dimensionless inequalities are computed by dividing the first three variables by the last one, $B L$, as shown below:

$$
\begin{gathered}
\left(\frac{\ell_{\max }}{B L}\right)^{2} \leq\left(\frac{1}{2} \frac{C L}{B L}\right)^{2}+\left[\frac{\left(3-\frac{p}{2}\right) \frac{C L}{B L}-1}{2-p)\left(\frac{W}{B L}\right) /\left(\frac{H}{B L}\right)}\right]^{2}, \\
\left(\frac{\ell_{\max }}{B L}\right)^{2} \leq\left(\frac{1}{2} \frac{C L}{B L}\right)^{2}+\left[\frac{\frac{p}{2} \frac{C L}{B L}-1}{(2-p)\left(\frac{W}{B L}\right) /\left(\frac{H}{B L}\right)}\right]^{2}, \\
\left(\frac{\ell_{\max }}{B L}\right) \geq \frac{1}{2}\left(\frac{C L}{B L}\right)^{2} /\left(\frac{W}{B L}\right) .
\end{gathered}
$$

Both interference scenarios indicate that $\ell_{\max } / B L$ is smaller than a specific value with given $W, H, B L$, and $p$. This means interference only limits the upper bounds of $\ell_{\max }$. As for "which case" binds the extreme value of $\ell_{\max }$ depends on the given values of $W, H, B L$, and $p$. In general, values of $H$ and $\ell_{\max }$ are usually smaller than one half of $B L$.

The inequalities shown in Equations (14)-(16) are plotted in Figure 4(a), where the coordinates of three axes are dimensionless $W / B L, H / B L$, and $\ell_{\max } / B L$. The volume enclosed by these three surfaces indicates feasible relations among these variables for successful 4-leg coordination. With the selections of $\ell_{\max }=21 \mathrm{~cm}$, $B L=44.4 \mathrm{~cm}$, and $p=42 \%$, the $2 \mathrm{D}$ cross-section can be extracted from the 3D plot, as shown in Figure 4(b), which is very informative in selecting suitable variables, such as $B L$ with given $W$ and $H$. It is important to note that the simplification from Equations (8)-(10) to Equations (11)-(12) is based on the assumption of $C L<B L<2 C L$. Different assumptions will yield different results, which further derives different inequalities. In the current setting the parameters $D_{a}$ are around $25.4 \%$, which leaves room for tuning $B L$ with respect to $W$ and $H$. If $D_{a}$ reaches its maximum value (i.e. half of the $C L$ ), the swing of the front and hind leg pairs occur consecutively, and the body length is strictly confined to $B L(n-0.5)$, where $n$ is a positive integer.

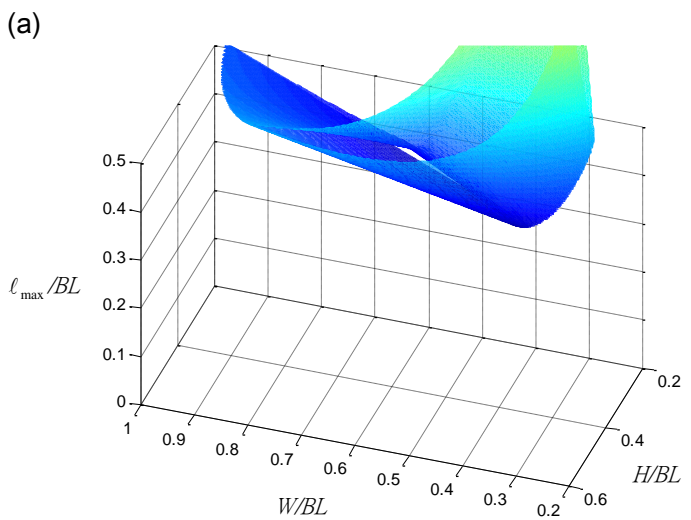

(b)

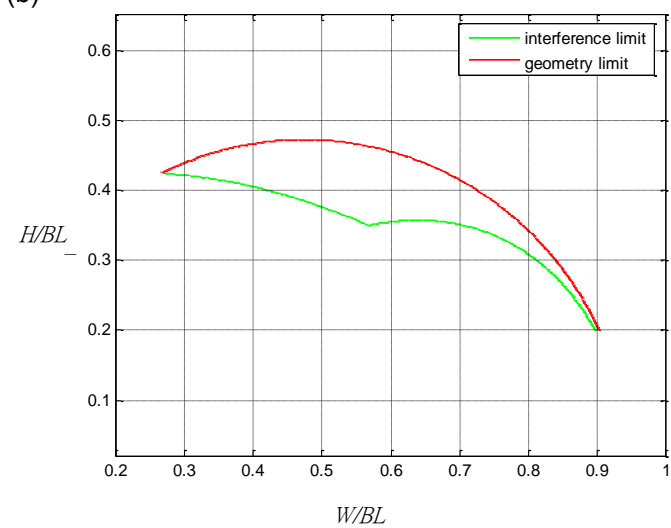

Figure 4. (a) The area bound by three surfaces indicates the feasible selection of dimensions which satisfies the inequality constraints Equations (14)-(16). (b) The feasible relative dimensions of $\mathrm{W}$ and $\mathrm{H}$ to $\mathrm{BL}$ is bound by three curves derived in Equations (14)-(16).

\section{Trajectory planning procedure}

The analysis thus far can be summarized in a standard design procedure, as described below:

1. With given sizes of stairs ( $W$ and $H$ ), the required minimum leg length $\ell_{\text {min }}$ can be computed according to Equation (1).

2. Assign the maximum leg length $\ell_{\max }$ according to Equations (14)-(16), avoiding the front and hind legs operating in the swing phase at the same time.

3. Define the desired hip clearance $d$.

4. Derive the contact offset $a$ of the right leg, $a_{R}$, from (4). Here we assume the right leg swings at its earliest possible timing, where the leg length is equal to $\ell_{\min }$ and $a_{R}$ is equal to $a_{\min }$.

5. Derive the contact offset $a$ of the left leg, $a_{L}$, from Equation (3), with $\ell$ equal to $\ell_{\max }$. Here we assume the left leg swings at its latest possible timing, where the leg length is equal to $\ell_{\max }$ and $a_{L}$ is equal to $a_{\max }$.

6. Set the parameter for 4-leg coordination according Equations (5)-(7).

With the above settings, the algorithm can be executed correctly. 


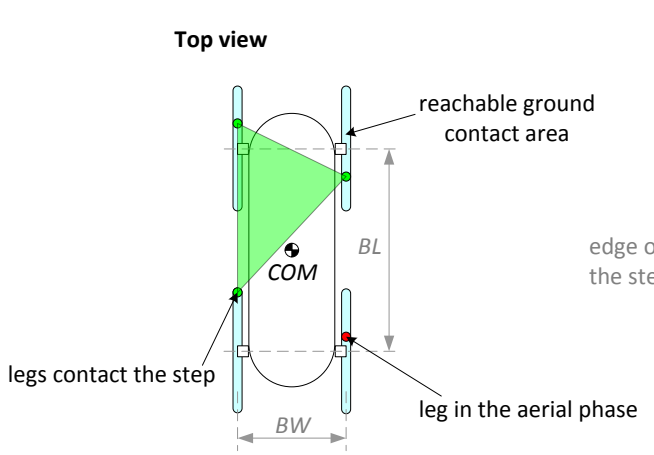

(a)

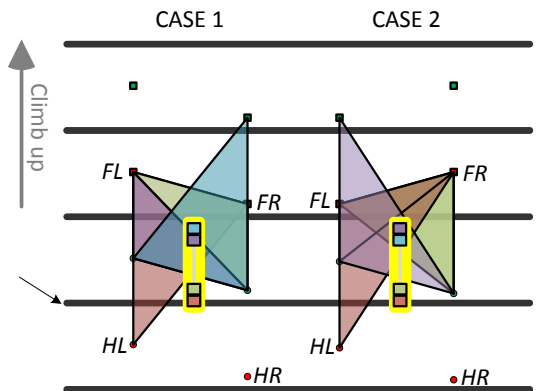

(b)

Figure 5. Quasi-static stability analysis of the robot: the geometrical relation between COM of the robot and the triangle formed by three stair-contact legs.

\section{Stability Analysis}

The above analysis is focused on motion planning on the sagittal plane. In practice, roll balance should be considered as well, especially in the situation where the robot is supported by less than four legs. Figure 5(a) shows that there exist certain moments when the COM might fall out of the instantaneous triangle region formed by three ground contact points, so the contact points of the legs with adequate contact offset $a_{i}$ on the stairs should be chosen carefully. Based on the permutation of the legs shown in Figure 5(b), two possible arrangements of $a_{i}$ are found. In the first case, both right legs contact the step with $a_{\min }$, where leg lengths are equal to $\ell_{\min }$, shown in Figure $2(b)$, and both left legs contact the step with $a_{\max }$, where the leg lengths are equal to $\ell_{\max }$. This is the scenario used in the previous sections. In this case, the swing sequence of all four legs in one period is $L_{H R}, L_{H L}, L_{F R}$, and $L_{F L}$. In the other case, $\left(a_{i}\right)$ s are switched in the front leg pairs. Thus, the swing sequence of all four legs in one period is $L_{H R}$, $L_{H L}, L_{F L}$, and $L_{F R}$. The quasi-static stability of the robot during stair climbing can be analyzed by the relative location of the COM with respect to the contact triangles formed by three step-contact legs, as shown in Figure 5. In both cases, each triangle indicates the instant where one specific leg is in swing phase, and the square mark in the same color as the triangle shows the position of the COM in that instant. This figure clearly shows that the robot would keep balanced as the front legs swing from the lower step to the upper one, but it may lose balance as the hind legs swing, since the COM falls out of the contact triangle. By using the same set of parameters $B L$ $=44.4 \mathrm{~cm}, B W=36 \mathrm{~cm}, \ell_{\max }=21 \mathrm{~cm}, p=42 \%$, the percentages of time where the COM of the robot falls out of the contact triangle in cases 1 and 2 are $27.3 \%$ and $30 \%$, respectively. The actual pitching and rolling behaviors of the robot due to this unbalanced moment are further determined by its time duration and the dynamics of the robots.

\section{Error Adlaptation}

In empirical settings, the sizes of the stairs are generally different from one another. Thus, how the difference in stair dimension affects the performance of the algorithm should be addressed accordingly. Following the parameter settings developed in the previous sections, assume that $W$ and $H$ are the average sizes of the group of stairs and $\Delta W \& \Delta H$ are the differences with their average values. The dimension error of the stair is discussed in two settings:

In the first case, only the depth $W$ of the stair has error $(\Delta W \neq 0, \Delta H=0)$. Figure $6(\mathrm{a})$ shows that the error of depth changes the distance $d$ only. According to the geometric analysis in the previous section, the distance $d$ is inverse with touchdown distance $a$. If $\Delta W>0, d$ becomes larger, and the touchdown position of the leg on the next step is located closer to the edge of the stair (i.e., value $a$ becomes smaller). When the error is too large, the robot might lose its ground contact. In contrast, if $\Delta W<0, d$ becomes smaller, the touchdown position of the leg on the next step moves closer to the edge of the next step (i.e., value $a$ becomes larger). In addition, the leg may hit the next step during swing phase. The permissible error range can be calculated based on the geometric relationship:

$$
a>\Delta W>\frac{1}{H+D_{s} \sin \phi}\left(\begin{array}{l}
D_{s} H \cos \phi+D_{s} b \sin \phi \\
-D_{s} W \sin \phi-\frac{d}{\sin \phi} H
\end{array}\right),
$$

where

$$
D_{s}=\left[\frac{W}{H} \sqrt{\ell_{\max }^{2}-\frac{1}{4}\left(W^{2}+H^{2}\right)}-\frac{1}{2} \sqrt{W^{2}+H^{2}}\right]
$$




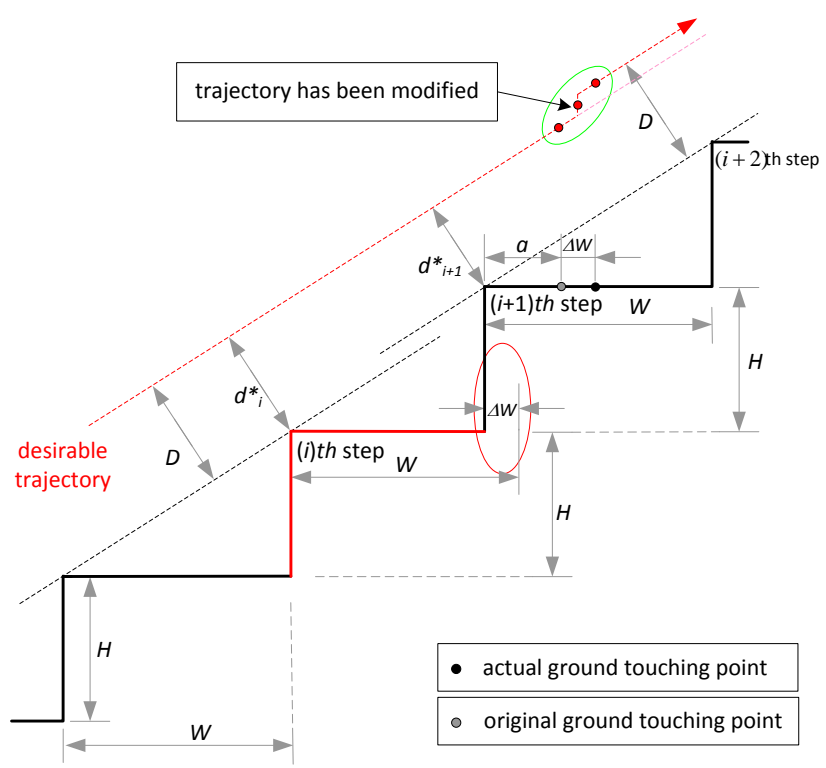

(a)

Figure 6. Schematic diagrams of the stair error correction.

is derived from (6). By using the same set of parameters, $W=27 \mathrm{~cm}, H=17 \mathrm{~cm}, \ell_{\max }=21 \mathrm{~cm}$, and $d=2.8 \mathrm{~cm}$, the acceptable range is $2.9>\Delta W>(-12.05 \mathrm{~cm})$. It is the overall limit of the error. Because error is accumulative, the computation should be made in an iterative manner. Assuming the robot moves on the $i^{\text {th }}$ stair, the $\Delta W_{t}$ is the summed errors generated by the previous stairs,

$$
\Delta W_{t}=\sum_{i=1} \Delta W_{i}
$$

If $\Delta W_{t}$ of all the steps are within the error range, the robot can operate safely during stair climbing without any sensory feedback and posture adjustment.

In the second case, only the height $H$ of the stair has error $(\Delta W=0, \Delta H \neq 0)$. If $\Delta H>0$, the leg may touch the stair in its swing phase, and the robot should calculate this $(\ell, \theta)$ immediately according to the new geometric relationship. This will satisfy the trajectory of the robot. If $\Delta H<0$, the leg may not be able to touch the step in its ground phase, and the swing phase should be prolonged. Empirically, the PD errors of the motor controls sensed by encoders can be utilized as the indicators of the ground contact condition.

\section{Simulation}

When the trajectories of the hips are known, the essential climbing parameters can be generated directly through a geometric relationship, including the timing of the swing phase, the touchdown distance $a_{R}$ and $a_{L}$ and $(\ell, \theta)$ of each leg. The algorithm developed in the

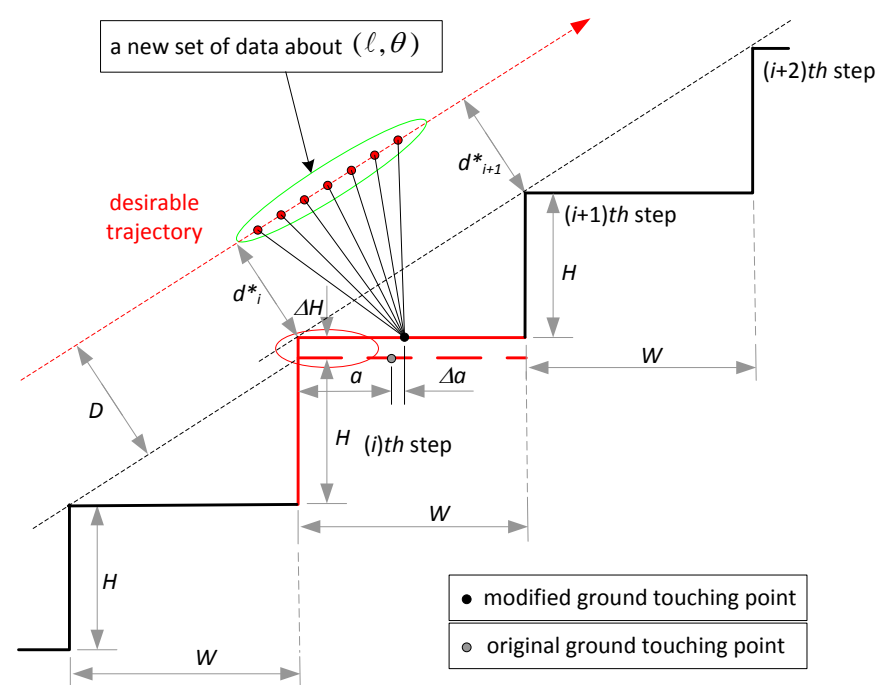

(b)

previous sections has been simulated in Matlab with a particular set of parameters $(W=27, H=17, d=8.5, L=$ $21, B L=44.4$, and $p=41 \%)$, which matches the parameters of Quattroped [14] and of general local stairs. Figure 7 (left) shows sequential snapshots of simulated results for the climbing of one step. The full simulation is available as the supplemental material associated with this paper. The simulation shows that the legs can be coordinated to swing from the lower step to the upper step in sequence with one travel distance $C L$ of COM without any interference, as expected from the analysis.

\section{Experiment Results and Discussion}

The algorithm was implemented on the robot Quattroped and experimentally evaluated. The robot climbed 10 times on two different-size stairs with geometry $(W=31 \mathrm{~cm}, H=17.5 \mathrm{~cm})$ and $(W=28 \mathrm{~cm}, H=$ $16 \mathrm{~cm}$ ). Please note that both sizes are different from the default settings $(W=27 \mathrm{~cm}, H=17 \mathrm{~cm})$, so the experimental runs were at a certain level testing the applicable size of stairs as well. Figure 7 (middle \& right) shows the sequential images extracted from one of the typical runs from each. The full video is also available as supplemental material associated with this paper. The two sets of 14 snapshots of the robot configuration also correspond to the 14 simulated subplots shown in Figure 7 (left) for comparison. Empirical evaluation reveals that the success rate is affected by the dimensions of the stairs, the ground contact condition, and the number of steps. The first two factors are the physical effects within the single step, and the last factor accumulates the 

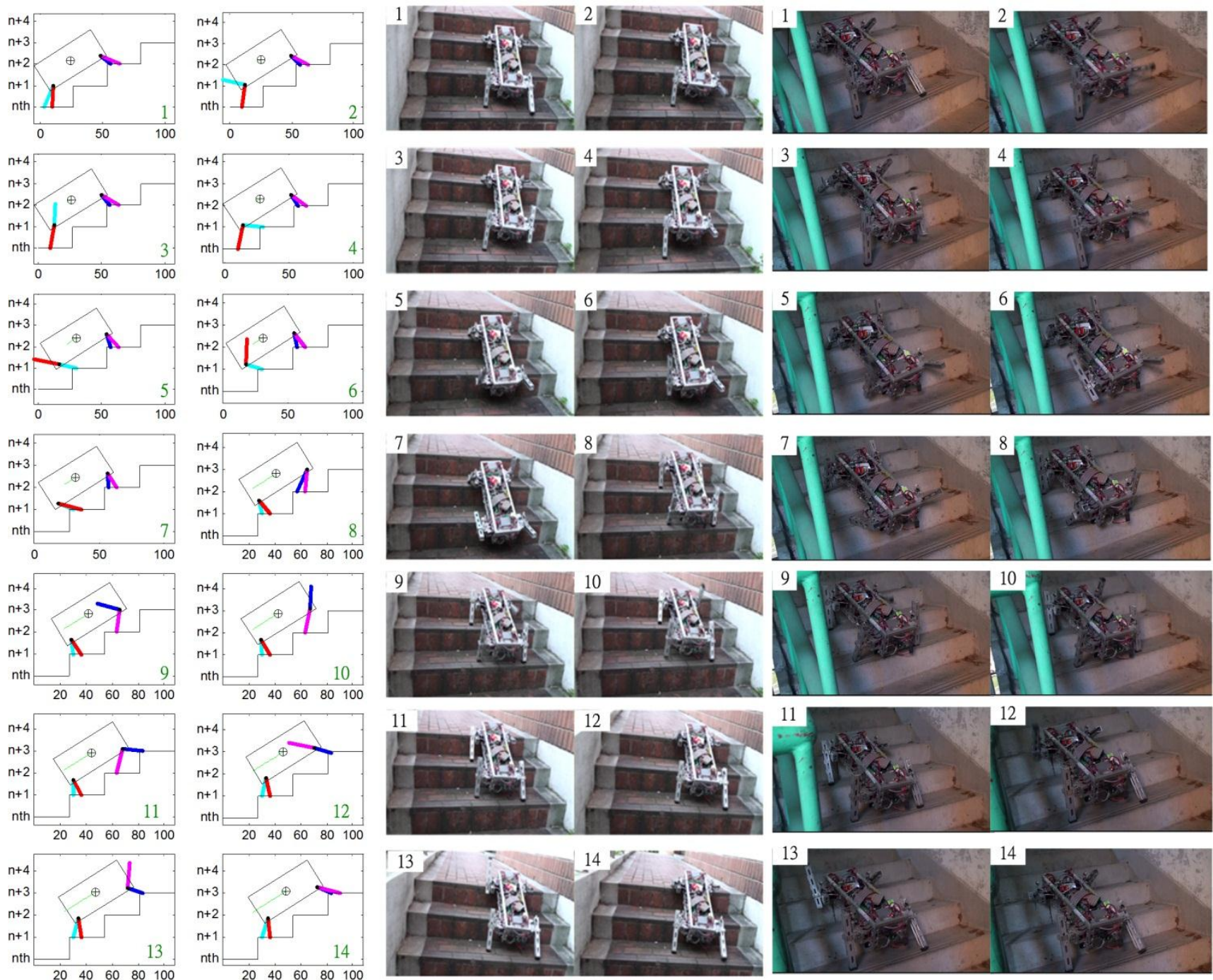

Figure 7. Sequential snapshots of the robot climbing the stair in a simulated and in physical environments. Left: The robot body and its COM are plotted as a rectangle and a cross within a circle. The magenta, blue, cyan, and red colors indicate front right, front left, hind right, and hind left legs, respectively. Unit of horizontal axis: $\mathrm{cm}$, unit of vertical axis: number of steps. Middle and right: The robot climbs the stairs with different geometrical parameters.

effects from the first two. When the robot climbs on the stairs with a size different than the default one, the timing and the position of the contact offset $a_{i, i=R, L}$ change. Because the change is bi-directional (increase or decrease), the robot is still capable of climbing up as long as the legs can swing onto the next stair. The drawback was the large leg tracking errors owing to the incorrect closed 4 bar linkage formed by fore-leg, body, hind-leg, and the stair. In contrast, the friction condition had a more severe effect than the stair dimensions. Unlike other locomotion tasks, stair climbing involves large potential energy generation which requires solid ground contact so the motor can generate effective leg motion to push the body up. If the stair is dirty or the surface is slippery, the friction force may not be sufficient to grant the contact in the static region. When slippage happens, the contact offset $a_{i}$ always moves toward edge of the step (i.e., introducing shallow contact) and the hip clearance $d$ increases gradually. After multiple-step climbing, the body may pose too far for the front leg to catch the stair, and the robot falls in this scenario. The step shown in Figure 7 (middle) has 7 steps, and the robot could climb with $100 \%$ successful rate. The step shown in Figure 7 (right) has more than 10 steps, and the robot usually falls after climbing 7-9 steps. Empirically the dimensions and the materials of the stairs always vary simultaneously. It is unrealistic to evaluate both factors individually. Instead, it is attractive to develop an adequate feedback mechanism which is capable of correcting the robot posture and maintaining the robot configuration relative to the stair. We are currently developing such an algorithm and the results will be reported separately. Following the logic in this paper, the paper's main contribution lies in the framework for designing the leg trajectory and coordination scheme for periodic locomotion like climbing stairs, and 
experimental results confirm that the algorithm with open-loop strategy is indeed functional and the robot can indeed climb the stairs with certain geometrical variations.

\section{Conclusion and Future Work}

We reported on the algorithm of trajectory planning and four-leg coordination for quasi-static stair climbing in a quadruped robot. The detailed development is based on the geometrical interactions between the robot legs and the stair. The suitable dimensions of the robot and how these parameters affect the algorithm are demonstrated. In addition, a brief study on the quasi-static stability of the robot shows that stability can usually be maintained, and possible unstable postures can be corrected, using the stable four-leg supporting posture. Finally, the algorithm is simulated and evaluated via experimentation, which confirms that the proposed algorithm is functional.

We are currently in the process of developing a feedback mechanism for the algorithm, which will further tolerate much wider geometrical variations of stairs. In the meantime, the dynamics of the system are under investigation.

\section{Acknowledgement}

This work is supported by National Science Council (NSC), Taiwan, under contract NSC 97-2218-E-002-022. The authors gratefully thank the National Instruments Taiwan Branch for their kind support with equipment and technical consulting.

\section{References}

[1] T. Estier, Y. Crausaz, B. Merminod, M. Lauria, R. Piguet, and R. Siegwart, "An innovative space rover with extended climbing abilities," ASCE Conference Proceedings, vol. 299, no. 40476, pp. 44-44, 2000. doi: $10.1061 / 40476(299) 44$

[2] S. D. Herbert, A. Drenner, and N. Papanikolopoulos, "Loper: A quadruped-hybrid stair climbing robot," in IEEE International Conference on Robotics and Automation, Pasadena, CA, 2008, pp. 799-804. doi: 10.1109/ROBOT.2008.4543303

[3] J. B. Jeans and D. Hong, "IMPASS: Intelligent mobility platform with active spoke system," in IEEE International Conference on Robotics and Automation, Kobe, Japan, 2009, pp. 1605-1606. doi: 10.1109/ROBOT.2009.5152735

[4] iRobot Corporation. Irobot: Packbot. Available: www.irobot.com
[5] American Honda Motor Co. Inc. History of ASIMO robotics.

Available: http://asimo.honda.com/asimo-history/

[6] P. Tantichattanont, S. Songschon, and S. Laksanacharoen, "Quasi-static analysis of a leg-wheel hybrid vehicle for enhancing stair climbing ability," in IEEE International Conference on Robotics and Biomimetics, Sanya, China, 2007, pp. 1601-1605.

doi: 10.1109/ROBIO.2007.4522404

[7] U. Saranli, M. Buehler, and D. E. Koditschek, "RHex: A simple and highly mobile hexapod robot," The International Journal of Robotics Research, vol. 20, no. 7, pp. 616-631, 2001. doi: $10.1177 / 02783640122067570$

[8] E. Z. Moore, D. Campbell, F. Grimminger, and M. Buehler, "Reliable stair climbing in the simple hexapod 'RHex'," in IEEE International Conference on Robotics and Automation, Washington, DC, 2002, pp. 2222-2227. doi: 10.1109/ROBOT.2002.1013562

[9] D. Campbell and M. Buehler, "Stair descent in the simple hexapod 'RHex'," in IEEE International Conference on Robotics and Automation, Taipei, Taiwan, 2003, pp. 1380- 1385 vol.1381-1380- 1385 vol.1381. doi: $10.1109 /$ ROBOT.2003.1241784

[10] I. Poulakakis, E. Papadopoulos, and M. Buehler, "On the stability of the passive dynamics of quadrupedal running with a bounding gait," The International Journal of Robotics Research, vol. 25, no. 7, pp. 669-687, 2006. doi: $10.1177 / 0278364906066768$

[11] H. Kimura, Y. Fukuoka, and A. H. Cohen, "Adaptive dynamic walking of a quadruped robot on natural ground based on biological concepts," The International Journal of Robotics Research, vol. 26, no. 5, pp. 475-490, 2007. doi: $10.1177 / 0278364907078089$

[12] K. Kato and S. Hirose, "Development of the quadruped walking robot, TITAN-IX - mechanical design concept and application for the humanitarian de-mining robot," Advanced Robotics, vol. 15, no. 2, pp. 191-204, 2001. doi: $10.1163 / 15685530152116227$

[13] M. A. Jimenez and P. G. d. Santos, "Attitude and position control method for realistic legged vehicles," Robotics and Autonomous Systems, vol. 18, no. 3, pp. 345-354, 1996. doi: 10.1016/0921-8890(95)00090-9

[14] S. Y. Shen, C. H. Li, C. C. Cheng, J. C. Lu, S. F. Wang, and P. C. Lin, "Design of a leg-wheel hybrid mobile platform," in IEEE/RSJ International Conference on Intelligent Robots and Systems, St. Louis, 2009, pp. 4682-4687. doi: $10.1109 /$ IROS.2009.5353958 